\title{
DENTISTRY IN MEDICINE
}

\author{
By F. W. E. WAGNer, M.A., Sc.D. \\ Fellow of the Royal Academy of Medicine of Ireland. Fellow of the Royal Society of \\ Tropical Medicine
}

\section{II.' Septic Conditions and Methods of Treatment}

Sometimes a patient arrives at the dentist's surgery and blithely informs the practitioner that 'Dr. Scalpel says I've got to have all my teeth out.' The dentist examines the mouth and finds a chronic general periodontitis which, in his judgment will respond to treatment. What is he to do ? Is he to do violence to his professional conscience and judgment by extracting all the teeth or is he to inform the patient that Dr. Scalpel is wrong, and that the septic condition will respond to treatment, thereby making Dr. Scalpel an enemy for life? It is a very difficult and a very uncomfortable position in which to find oneself, and it is a common enough experience. So I begin this article with a plea to medical practitioners not to refer patients to the dentist in this way. The dentist is a specialist in dental disease, and it is not much use referring a patient to a specialist with a definite instruction as to the treatment he is to adopt. How much pleasanter it is when a patient says, 'Dr. Scalpel has advised me to have a dental opinion about the state of my mouth, whether you think it can be treated or whether the teeth should come out.' In my previous article I referred to the policy of temporizing with acute conditions; and I feel that I must say a little more about it in connection with the treatment of septic and inflammatory conditions such as chronic periodontitis, alveolar abscess, antral empyema, etc. Even in these days one finds medical practitioners laying down the dictum that this or that tooth or teeth must not be extracted until the swelling has gone down or the septic conditions have subsided. To revert to my illustration of last month, when they have to deal with an acute appendix they rightly have the patient on the operating table with all possible speed, so why not do the same thing with acute teeth ?

It would avoid the unpleasant dilemma in which we find ourselves when a patient comes in with alveolar abscess or. an inflamed impacted third molar and tells us, 'Dr. Scalpel . says I mustn't have these out until the swelling has gone down.' We know quite well that the sooner we get that patient into the chair, remove those teeth under a general anaesthetic and establish drainage the better it is for our patient. I plead that there shall be discarded that erroneous idea (it seems, like the Merry Monarch, to be 'an unconscionable time in dying ') that you must not operate on teeth while septic conditions are acute. It is true that you must not operate in such conditions with a local anaesthetic, but that is all.

Iet us consider one of the commonest septic conditions met with in practice, Vincent's Infection. I stress this because the medical practitioner probably sees far more cases of this disease in its early stages than does his dental colleague, and if medical practitioners would either treat it themselves or refer it to us for treatment, it would go a long way towards reducing the incidence of a disease which is menacing the health of the community. Our trouble is that we seldom see these cases until they are in advanced stages.

I know of no better description of the clinical appearances in a typical case than that given by Colyer and Sprawson, so I propose to. quote it in extenso.

'In the early stages the gums are swollen and congested. The inflammation and ulceration commence at the free margin of the gums, these, when ulcerated, bleed readily and present a ragged margin of necrotic tissue, the infection spreads rapidly and may within twenty-four to forty-eight hours involve both jaws, and possibly extend to the hard palate and to the floor of the mouth, the adjacent mucous surface of the cheek may become attacked, possibly from contact, and in untreated cases the extension of the process may lead to extensive sloughing, necrosis of bone, 
and loosening and loss of tecth and even the death of the patient. The surfaces of the ulcers are covered with yellowish or greerishyellow sloughs, which readily become detached exposing a freely-bleeding surface, the ulcerated surface may remain superficial or extend more deeply and lead to exposure and ultimate necrosis of bone. The breath is very offensive and there is a rise of temperature from 100 to 103 , the lymphatic glands draining the area are enlarged.' (Dental Surgery and Pathology, 6th ed., p. 465.)

The main principle of treatment is to attack the disease both locally and systemically. First of all any deposits on the teeth should be removed carefully, gently and thoroughly. If the disease is in the early stages instruct the patient to cleanse the tceth and gums night and morning with warm water and common salt, and then to spend five minutes by the clock washing out the mouth with a mixture of half perovide and half hot water. As adjuvant to this the patient may allow a 5-gr. tablet of potassium chlorate to dissolve slowly in the mouth three or four times a day. This simple treatment will prove adequate for most cases.

But if the disease is further developed and has really taken hold, then prepare a paste from two or three crushed tablets of sulphathiazole and sulphathiazole ointment, and pack this paste into pockets, under loose gum margins and into interdental spaces back and fiont. This should be done daily for three or four days and then on alternate days until the disease has subsided. At the same time the patient should be put on a course of sulphathiazole -an initial dose of $4 \mathrm{~g}$. then $\mathrm{I}$ g. every four hours for seven days ; thereafter dosage as clinically indicated. When the healing of the tissues is complete the patient should use the following tooth-powder as a prophylactic measure :-

R

Mag. Perhydrol.

Sulphathiazole.

Pulv. Orris Root.

Pulv. Calcium Carbonat. ppt.

Menthol.

Ol. Peppermint.

Saccharin. Solubil.

gr. iv.

gr. iii.

$\xi \mathrm{i}$.

$\overline{3}$ iss.

gr. v.

m. vii

gr. iv.

Use as a tooth-powder and, mixed with a little water as a mouthwash night and morning.

If there is difficulty in obtaining Mag.
Perhydrol. then Mag. Parox. can be substituted. If Orris Root cannot be obtained increase calcium carbonate. Other successful methods of treatment are local application of Sulpharsphenamin on a swab moistened with Glycerin ( 1 in 15 ). This may also be injected, $0.18 \mathrm{~g}$. intramuscularly or subcutaneously; or 50 to $250 \mathrm{mg}$. of Nicotinic Acid may be prescribed daily for a week.

Alveolar abscess or 'gumboil' is a faitly common complaint, and one that a medical practitioner is often asked to treat. If it is accompanied by a sinus there is not usually much pain. The abscess periodically fills and discharges. If it is not accompanied by a sinus treatment is 1ather more urgent. Any application of heat should be inside the mouth, not outside. The evil practice of poulticing the cheek usually results in the abscess perforating the cheek and leaving a scar. Extraction of the offending tooth under a general anaesthetic is the most satisfactory tieatment. If it is particularly desired to save the tooth it will be necessary to open up the root canal, remove the pulp and, after repeated dressing and making every possible effort to sterilize the root canal, filling it. But this is always more or less a gamble. After taking the utmost care one often finds that an apical abscess develops, and then the tooth must be removed. If a patient specially wishes me to save the tooth, I point out the risk involved and leave it to the patient to decide. I doubt if I succeed in more than about half the cases I attempt and, frankly, I do not think it is worth while. The dangers of dental sepsis are considerable, and I think that extraction is the better treatment. A neglected alveolar abscess may lead to osteomyelitis. Thrush, which is characterized by grey-white spots on the mucous membrane of the mouth, which spots coalesce into patches which peel away leaving a bleeding surface is a disease which occurs in children fed from a dirty bottle. It sometimes occurs in debilitated adults, but mostly we see it in children. Particular care must be taken to keep feeding utensils clean. The treatment is simple; the mouth must be well wiped out before and after food with clean lint dipped in Glycerin of Borax. In very resistant cases the mouth should be swabbed with a solution of Sodium Sulphite, I dr. to 2 ozs. of water, before apply- 
ing the Glycerin of Borax. The same treatment may be used for adults.

Antral inflammation may be either acute or chronic. In the former type there is severe and throbbing pain deep in the cheek, and this is increased if the patient leans forward and bends down. Pain can be elicited by pressure on the malar bone or the canine fossa. It is more frequently caused by intra-nasal than by dental infection. If it is caused by a tooth, that tooth will be tender and painful when it is subjected to pressure or percussion ; and such a tooth should be removed. As a rule the first symptom of which the patient complains is a foul discharge from the nostril on the affected side, or po sibly a similar discharge into the naso-pharynx. The discharge affords relief from pain.

When the tooth has been removed, the next step is to establish free communication with the antrum through the alveolus. The communicating passage should be of adequate size, sufficient to admit a plug of 2 or $3 \mathrm{~mm}$. in diameter. The plug should be solid rather than a tube, as the tube tends to get choked with food or septic matter. I have found the Tilley vulcanite plug very satisfactory. During the first three days following operation the plug should be removed three times a day and the antrum washed out with warm normal saline solution. Then twice a day will be enough, and when no pus has appeared or been washed out for four or five days the tube can be discarded, but the washing out of the antrum should be continued until the communicating passage has closed up. As a rule it takes anything from a fortnight to three weeks to effect a cure.

Pain is not a very marked symptom of chronic inflammation unless there is some obstruction hindering the outlet of pus from the antrum. Catarrhal symptoms are frequently met with. General debility is not a 1 uncommon accompaniment, and is due in part to the constant absorption of toxins.

A dead tooth or a crowned tooth or even a filled tooth should be very carefully examined. If this is the cause or contributory cause it should be removed, but more radic al treatment than alveolar drainage-which usually fails in chronic cases-is called for. The opinion of the oto-laryngologist is then most necessary.

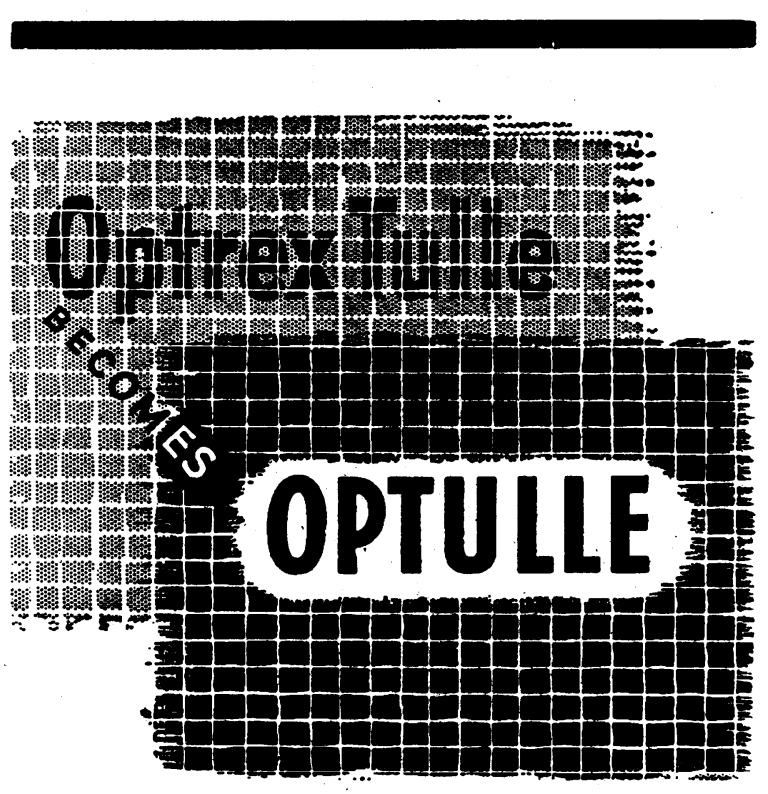

For the sake of convenience, we have decided to change the name of our increasingly popular gauze dressing from “Optrex Tulle" to "Optulle". The dressing itself, however, has not been altered in any way. It remains exactly the same $-a$ wide mesh gauze impregnated with Balsam of Peru in a greasy base - suitable for a wide variety of uses, particularly for raw surfaces, including burns and septic wounds, and for plastic surgery cases.

Supplied in tins of 24 dressings, 4 ins. square (approx.) Prices to the Medical Profession 4/-per tin or 45/-perdozen.

MANUFACTURED BY

OPTREX LTD., PERIVALE, midDleseX

Sole Distributors: CHAS. F. THACKRAY LTD. THE OLD MEDICAL SCHOOL, PARK ST., LEEDS, I also LONDON AND CAPE TOWN 